\title{
Hábitos saludables en universitarios de ciencias de la salud y de otras ramas de conocimiento: un estudio comparativo
}

\author{
Alba Solera Sánchez ${ }^{a}$, Amparo Gamero Lluna ${ }^{a, b, *}$

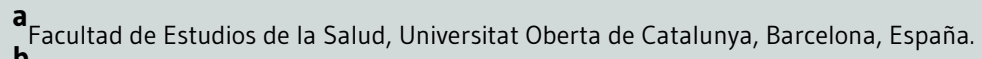 \\ bepartamento de Medicina, Preventiva y Salud Pública, Ciencias de la Alimentación, Toxicología y Medicina Legal, \\ Facultad de Farmacia, Universitat de València, España. \\ *amparo.gamero@uv.es
}

Editor Asignado: Eduard Baladia. Comité Editorial de la Revista Española de Nutrición Humana y Dietética. Pamplona, España.

Recibido el 1 de marzo de 2019; aceptado el 5 de diciembre de 2019; publicado el 19 de diciembre de 2019.

Hábitos saludables en universitarios de ciencias de la salud

y de otras ramas de conocimiento: un estudio comparativo

\section{PALABRAS CLAVE}

Conducta

Alimentaria;

Estudiantes;

Dieta Mediterránea;

Ejercicio;

Salud.

\section{RESUMEN}

Introducción: Durante la vida universitaria, los estudiantes deberían consolidar sus hábitos saludables, que se verán reflejados posteriormente en su vida adulta. Esto es de especial importancia en el caso de los estudiantes que lleguen a ser profesionales del ámbito de la salud. Sin embargo, gran parte de la literatura concluye que los estudiantes reportan malos hábitos durante el periodo universitario. Objetivos: El presente estudio pretende determinar si estudiantes de ciencias de la salud (ECS) y estudiantes de otras ciencias (EOC) presentan hábitos saludables relacionados con la práctica de actividad física (AF) y adherencia a la Dieta Mediterránea (DM). Asimismo, saber si los ECS tienen hábitos más saludables que los EOC.

Material y Métodos: Se evaluaron los hábitos saludables de 104 estudiantes (52 ECS y 52 EOC) con edades comprendidas entre los 19 y 32 años. La práctica regular de AF fue determinada con el Cuestionario Internacional de Actividad Física (IPAQ) y la adherencia a la DM, juntamente con los hábitos nutricionales, con el cuestionario PrediMed.

Resultados: Un 51,92\% de los ECS reportaron una práctica de AF relacionada principalmente con actividades vigorosas, mientras que el resto, con moderadas y leves. Por el contrario, la mayoría de EOC (67,31\%) tiende a practicar AF leve. En relación con la adherencia a la DM, un 54\% de los ECS presentaron adherencia a la DM en contraste con sólo un $27 \%$ de los EOC. Finalmente, los ECS presentaron un consumo más elevado de frutas y zumos naturales, pescados y mariscos y frutos secos, mientras que los EOC se decantaron por un consumo mayor de carnes rojas y procesadas y bebidas azucaradas.

Conclusiones: Los ECS presentaron hábitos más saludables que los EOC, una práctica regular de AF más intensa y una mayor adherencia a la DM. Se demuestra una relación directa entre los conocimientos en salud y unos hábitos más saludables en la población universitaria. 
Healthy habits of health sciences students and others from different fields: a comparative study

\section{KEYWORDS}

Feeding Behavior;

Students;

Diet, Mediterranean;

Exercise;

Health.

\section{ABSTRACT}

Introduction: During the university life, students should consolidate their healthy habits, which should eventually be reflected in the adult life. This is of special importance in case of students who will become professionals of health field. However, lots of investigations conclude that students present really bad habits during their period at university. Objectives: The present study aimed to determine if students from health sciences (ECS) and students from other sciences (EOC) present healthy habits related to regular physical activity practice (AF) and adherence to Mediterranean Diet (DM). In addition, if the ECS have healthier habits than the EOC was investigated.

Material and Methods: Healthy habits were evaluated in a sample of 104 students (52 ECS and 52 EOC) from 19 to 32 years old. The AF was determined with the International Physical Activity Questionnaire (IPAQ) and the adherence to DM and the nutritional habits, employing PrediMed Questionnaire.

Results: $51.92 \%$ of ECS reported an AF practice mainly related to vigorous activities whereas the remaining ones, to moderate and light. On the contrary, most of the EOC use to practice light AF. Regarding the adherence to the DM, 54\% of ECS presented adherence to DM in contrast to only $27 \%$ of EOC. Finally, the ECS had a greater consumption of fruit and natural juices, fish and seafood and nuts, while the EOC reported greater consumptions of red and processed meat and sugar beverages.

Conclusions: The ECS reported healthier habits than the EOC, a regular practice of more vigorous AF and higher adherence to DM. A direct relationship between health knowledge and a healthier habit among university population is proved.

\section{CITA}

Solera Sánchez A, Gamero Lluna A. Hábitos saludables en universitarios de ciencias de la salud y de otras ramas de conocimiento: un estudio comparativo. Rev Esp Nutr Hum Diet. 2019; 23(4): 271-82. doi: 10.14306/ renhyd.23.4.762

\section{INTRODUCCIÓN}

Los hábitos de vida saludables hacen referencia a patrones de comportamiento que incluyen una alimentación equilibrada y con pocos productos procesados, una hidratación adecuada, y la práctica de actividad física (AF) regular ${ }^{1}$. Llevar una alimentación saludable es una práctica relacionada con un aumento de la esperanza de vida, mejores parámetros metabólicos, mantenimiento de la salud cardiovascular y salud psicológica de los individuos².
La AF o deportiva regular representa un pilar básico en el mantenimiento de hábitos de vida saludables. Según Escámez et al. ${ }^{3}$, la práctica periódica de AF es un factor de prevención de enfermedades como la hipercolesterolemia, hipertensión, diabetes, cáncer, osteoporosis y el dolor crónico de espalda, además de producir efectos beneficiosos a nivel psicológico, como la mejora del estado de ánimo y la autoestima y la reducción del estrés y la ansiedad. Practicar algún tipo de AF o deportiva, según la evidencia científica, aporta a la persona beneficios en diferentes ámbitos vitales, como el físico, el psicológico, el socio-afectivo y el fisiológico ${ }^{4}$. 
El segundo pilar básico en la práctica de hábitos saludables es la alimentación. La adherencia a la Dieta Mediterránea (DM) representa un ejemplo de alimentación beneficiosa para la salud. Ésta se basa en el consumo de vegetales, frutas, hortalizas, aceite de oliva, legumbres, frutos secos, aceitunas, vino, yogur o queso, preferentemente de cabra y oveja, carnes blancas, pescado y con mucha moderación azúcares refinados, carnes rojas y procesadas ${ }^{5}$. La adherencia a este tipo de dieta tiene infinidad de efectos favorables para la salud, entre los que se encuentran la reducción de la prevalencia de enfermedades neurodegenerativas, la diabetes, las enfermedades cardiovasculares, diferentes tipos de cáncer e incluso el retraso del envejecimiento ${ }^{6}$.

A pesar de todos los beneficios que aporta la DM para la salud y la gran disponibilidad que se tiene de los alimentos que la componen gracias a la globalización, Cuervo et al. ${ }^{7}$ reportan la expansión de las cadenas de comida rápida debido a la gran demanda de la población, un aumento en el consumo de bebidas azucaradas y alcohólicas, mayor número de dietas inestables, hábitos nutricionales familiares inadecuados y la necesidad de comer fuera para desarrollarse socialmente. Estos factores provocan hábitos alimentarios deficientes y poca adherencia a la DM.

Las consecuencias de esta tendencia hacia los malos hábitos en la dieta pueden ser desastrosas para la salud, Ilevando a la población a padecer sobrepeso u obesidad y acabar provocando diversas enfermedades asociadas a ellos ${ }^{8}$.

Los riesgos de llevar prácticas alimentarias no saludables son altos en todos los segmentos de población. Kovesdy et al. ${ }^{9}$ concluyeron en que la obesidad está llegando a extremos en la que se la considera la epidemia a escala mundial y afecta a niños, adolescentes y adultos.

Una de las poblaciones con un alto riesgo de llevar hábitos no saludables son los jóvenes universitarios ${ }^{10}$. González et al. ${ }^{11}$ reportaron que un $17,1 \%$ de los sujetos que participaron en la investigación (424 estudiantes) presentaban sobrepeso según su índice de masa corporal (IMC). Por lo tanto, está claro que los estilos de vida actuales y las tendencias futuras no ayudan a la adopción de estilos de vida saludables en la población universitaria.

Según Joyce et al. ${ }^{12}$, el gran volumen de trabajo en estos estudiantes puede provocar una rutina cargada de inactividad física. Esto sucede ya que los trabajos universitarios de todas las ramas de conocimiento deben de ser de gran calidad e implicar rigurosas investigaciones y un valor añadido ${ }^{13}$. Estas características que debe de tener cualquier trabajo o actividad universitaria, representan mucha dedicación e implicación por parte del/la estudiante, tiempo que dedican a estar sentados en una silla con el ordenador y debido a ello llevan vidas con poca o mínima $\mathrm{AF}^{14}$. Además, todos ellos deben afrontar exigencias altas, continuas adaptaciones y retos que los pueden llevar a situaciones de estrés que impliquen este deterioro de hábitos saludables ${ }^{15}$.

El hecho de llevar un estilo de vida no saludable, y el riesgo de que estos hábitos se conserven durante la adultez, representa un gran inconveniente para ellos, pero sobre todo para los estudiantes relacionados con las ciencias de la salud (ECS). La promoción de hábitos saludables, como la AF y el mantenimiento de una buena alimentación, es una de las labores primordiales de estos futuros profesionales. Por ello, no es necesario que sólo conozcan las bases de estos hábitos, sino que también los practiquen ${ }^{16}$. Los ECS estudian para mejorar o mantener la salud de la población, por lo que adquieren muchos conocimientos que deberían convertirse en actitudes positivas que incentiven un compromiso y una conducta promotora de hábitos saludables para la sociedad ${ }^{17}$.

Considerando todo lo expuesto y teniendo en cuenta que existe mucha literatura sobre los hábitos poco saludables de los universitarios, pero poca diferenciando los ECS y los estudiantes de otras ramas de conocimiento (EOC), el presente estudio pretende comparar los hábitos saludables (adherencia a la DM, hábitos alimentarios y práctica de AF) de ECS y EOC, así como determinar si los primeros presentan mejores hábitos y promocionan esos estilos de vida como deberían hacer los futuros profesionales de la salud.

\section{MATERIAL Y MÉTODOS}

El trabajo de investigación fue descriptivo y transversal, ya que se describen los datos obtenidos en los cuestionarios que reflejan los hábitos saludables en un momento dado.

\section{Población}

Para la recogida de datos se escogieron de forma aleatoria 104 sujetos universitarios. Un $48 \%$ fueron hombres y un $52 \%$ mujeres. Las edades estaban comprendidas entre 19 y 32 años. Los estudiantes pertenecían a todos los cursos de grado y también hubo alumnos de máster. Los ECS fueron de ciencias de la actividad física y el deporte, fisioterapia y enfermería. Y los EOC fueron de criminología, psicología, derecho, física, relaciones laborales, economía, periodismo y comunicación. Los universitarios estudiaban en la Universidad de Vic, Universidad Autónoma de Barcelona y Escuelas Universitarias Gimbernat. Los periodos en los que los/las estudiantes rellenaron los cuestionarios 
fueron durante mayo y junio, época en la que tenían las entregas finales y exámenes y eso pudo afectar a los datos recogidos sobre la práctica habitual de AF.

\section{Variables de estudio}

El método utilizado fue la recolección de datos mediante dos encuestas a la población objeto de estudio. Las encuestas fueron presentadas a los sujetos presencialmente y en papel. Se explicaron las encuestas en persona a los sujetos y se resolvieron las dudas que pudieron surgir en el momento de la realización de las encuestas. Los datos que se recogieron fueron sobre la práctica de AF habitual con el cuestionario internacional IPAQ, la adherencia a la DM y los hábitos alimentarios a través del cuestionario PrediMed.

La encuesta IPAQ pretende mostrar la práctica total de AF de la población estudiada. El cuestionario está formado por 7 preguntas, ya que pertenece a la versión corta existente en la actualidad. Estas preguntas valoran la AF que el sujeto ha realizado durante los últimos 7 días (durante el tiempo libre y entrenamientos o en las actividades de la vida diaria), junto con la intensidad a la que se han realizado y el tiempo total de éstas. Mediante la encuesta se obtienen los minutos de AF leve, moderada y vigorosa que el sujeto ha practicado y se realizan los siguientes cálculos:

a) Actividad leve: 3,3 METS * minutos de actividad * días a la semana.

b) Actividad moderada: 4 METS * minutos de actividad * días a la semana.

c) Actividad vigorosa: 8 METS * minutos de actividad * días a la semana.

Con estas fórmulas se obtienen los minutos de AF que el sujeto ha realizado de cada intensidad y se expresan en METS/semana. Para obtener la AF total, se suman los METS/semana obtenidos para cada intensidad de actividad (leve, moderada y vigorosa). Finalmente, se clasifica la AF total realizada por el sujeto de la siguiente manera:

Leve: si los METS/semana son inferiores a 600.

Moderada: si los METS/semana son igual o mayores a 600.

Vigorosa: si los METS/semana son igual o superiores a 1.500.

Para confirmar la fiabilidad de los cuestionarios y poder utilizarlos asegurándonos datos objetivos, se ha hecho una búsqueda previa de estudios que los han validado o comprobado su fiabilidad ${ }^{18-20}$.

En este estudio se muestran los resultados para cada nivel de actividad (leve, moderada o vigorosa) antes y después de obtener la AF total y clasificarla.
El cuestionario PrediMed de adherencia a la DM consta de 14 preguntas que se tienen que responder mediante "sí" o "no" o mediante una escala del 1 al 3 o más de 3, según el consumo del grupo de alimentos que aparece en la pregunta. Se incluyó una respuesta de consumo 0 entre las posibilidades del test. Las preguntas que tienen respuestas acordes con hábitos correspondientes a la DM suman puntos. Y las que no van en la línea de los hábitos alimentarios asociados a la DM valen 0 puntos. La puntuación obtenida determina la adherencia de los sujetos:

1) 9 puntos o más: Existe adherencia a la DM.

2) 8 puntos o menos: Baja adherencia a la DM.

La frecuencia de consumo de alimentos se extrajo a partir del cuestionario PrediMed.

\section{Análisis estadístico}

Se realizó un análisis de regresión logística para determinar si las variables edad, sexo y tipo de estudios poseían una influencia positiva o negativa para la práctica de AF habitual y la adherencia a la DM. Más concretamente, en el caso de la AF se realizó un análisis de regresión logística ordinal, mientras que en el caso de la adherencia a la DM, un análisis de regresión logística binario. Para ello se empleó el programa XLSTAT (Microsoft, USA).

\section{RESULTADOS}

Tras la aplicación de las encuestas a los dos grupos objeto de estudio, ECS y EOC, se analizaron y compararon los resultados en cuanto a: (1) Práctica de AF habitual; (2) Adherencia a la DM; (3) Hábitos de consumo de los grupos de alimentos asociados a la DM.

\section{Práctica de AF habitual}

Respecto a la intensidad de la AF practicada, el modelo obtenido mediante análisis de regresión logística ordinal relaciona a los EOC con un nivel de AF leve o moderado, en contraposición a los ECS, que presentan mayoritariamente una $A F$ de intensidad vigorosa (verosimilitud del modelo 0,019).

En la Tabla 1 se detallan los porcentajes de sujetos de los dos grupos (ECS y EOC) que practican AF baja, moderada o vigorosa, según la metodología que marca el cuestionario IPAQ. Las unidades utilizadas son los METS/semana, según los resultados del cuestionario. 
Tabla 1. Porcentaje de sujetos por grupos que practicaban AF baja, moderada o vigorosa semanal.
AF leve
AF moderada
AF vigorosa
( $<600 \mathrm{METS} / \mathrm{s}$ )
( $\geq 600 \mathrm{METS} / \mathrm{s}$ )
( $\leq 1.500 \mathrm{METS} / \mathrm{s}$ )
ECS
$5,8 \%$
$5,8 \%$
$88,5 \%$
EOC
$13,5 \%$
$25 \%$
$61,5 \%$

ECS: Estudiantes de Ciencias de la Salud; EOC: Estudiantes de otras ciencias; METS/s: METS/semana.

En esta tabla se puede observar cómo el $88,5 \%$ de los ECS practicaban actividad vigorosa a la semana, debido a la suma de toda la AF de intensidad vigorosa, moderada y leve que resultaba en más de $1.500 \mathrm{METS} / \mathrm{s}$. Este resultado se encuentra por encima de las recomendaciones mínimas semanales de AF de la OMS (600 METS/semana).

Por el contrario, tan sólo un $61,5 \%$ de los EOC practicaban actividades físicas vigorosas. Se observa por tanto una diferencia significativa en la práctica de AF vigorosa entre los dos grupos. Además, vemos cómo sólo un $11,6 \%$ de los ECS practicaban AF leve o moderada frente a un $38,5 \%$ de los EOC, por lo que los EOC presentaron una mayor tendencia a la práctica de AF de menor intensidad.

Por otro lado, si se tiene en cuenta la AF que principalmente practicaba cada individuo y no la total semanal, la verosimilitud del modelo de regresión logística ordinal mejora hasta ser $<0,0001 ; y$ además de resaltar la relación de los EOC con AF de intensidad leve o moderada, se predice que a mayor edad menor será la intensidad de la AF practicada habitualmente por los estudiantes.

La Tabla 2 muestra resultados más específicos respecto a la intensidad de AF, ya que clasifica a los individuos de cada grupo (ECS y EOC) según el nivel de intensidad de AF que

Tabla 2. Práctica habitual de AF en estudiantes universitarios previa suma de AF total y clasificación según las indicaciones del cuestionario IPAQ.

$$
\text { Total sujetos que practican AF (\%) }
$$$$
\text { LEV : MOD : VIG }
$$

\begin{tabular}{l:l:l|l} 
ECS & 30,8 & 17,3 & 52,9
\end{tabular}

\begin{tabular}{l|l|l|l} 
EOC & 67,3 & 13,5 & 19,2
\end{tabular}

LEV: Actividad física leve; MOD: Actividad física moderada; VIG: Actividad física vigorosa; ECS: Estudiantes de Ciencias de la Salud; EOC: Estudiantes de otras ciencias. principalmente practicaban, expresando dicha actividad en METS semanales sin obtener la AF total y clasificarla. Según los datos, los ECS practicaban más AF vigorosa que moderada o leve al cabo de la semana. Concretamente, un $51,9 \%$ de Ios ECS practicaban AF vigorosa, frente al $17,3 \%$ y el $30,8 \%$ que la practicaban moderada y leve, respectivamente. Los EOC presentaban más práctica de AF leve $(67,3 \%)$ y sólo un $19,2 \%$ práctica de AF vigorosa.

De esta forma se puede observar que teniendo en cuenta las actividades de cada intensidad por separado (Tabla 2), sin obtener la AF total y clasificarla (Tabla 1), se obtienen resultados más cercanos a la realidad en cuanto a la intensidad de la AF realizada por cada grupo.

\section{Adherencia a la DM}

En cuanto a la adherencia a la DM, el análisis de regresión logística mostró que la variable EOC posee una contribución negativa en el modelo, de forma que la pertenencia a este grupo hace más probable una menor adherencia a la DM. Además, la edad o ser del sexo femenino también influyeron negativamente a esa adherencia (verosimilitud del modelo $<0,0001)$.

La diferencia entre los universitarios que presentaron adherencia a la DM (54\%) y los que no la presentaron (46\%) fue de un $8 \%$, siendo el porcentaje de adherencia a la DM ligeramente superior. Sin embargo, el 73\% de los EOC no presentaron adherencia a la DM. Si comparamos los dos grupos de estudiantes, vemos que la práctica de una buena nutrición acorde con las recomendaciones de la DM fue un $19 \%$ mayor en los ECS.

\section{Hábitos de consumo de los grupos de alimentos asociados a la DM}

El cuestionario PrediMed se basa en las frecuencias de consumo de los diferentes grupos de alimentos recogidas en la Tabla 3 para determinar si la adherencia a la DM es baja o adecuada. 
Tabla 3. Grupos de alimentos y su frecuencia de consumo en las dietas mediterráneas ${ }^{21}$.

Grupo de alimentos

Verduras

Frutas

Aceite de oliva

Cereales, principalmente pan y pastas

Legumbres

Frutos secos

Productos lácteos, principalmente fermentados, yogur y quesos

Huevos

Pescados y mariscos

Aves

Carnes rojas y procesadas

Vino

Especias y condimentos
Frecuencia de consumo

Diaria, en abundante cantidad, 3 o más porciones al día, crudas y cocidas

Diaria, en abundante cantidad, 2 o más porciones al día

Diaria, 3 a 6 cucharadas al día, como principal fuente de grasa

Diaria, 3 a 4 veces al día en moderada cantidad

Al menos 3 veces por semana

Al menos 3 veces por semana

Diaria, 2 a 4 porciones al día

1 a 4 unidades por semana

2 a 4 veces por semana

2 a 4 veces por semana

1 vez por semana o menos

Diaria, consumo moderado (1 copa al día para mujeres y 2 copas al día para hombres) y en forma regular, principalmente con las comidas

Diaria, uso habitual y variado en la preparación de las comidas
La Tabla 4 muestra los resultados más relevantes obtenidos mediante la aplicación del cuestionario PrediMed en la población estudiada, observándose hábitos distintos entre ambos grupos respecto a la frecuencia de consumo de ciertos grupos de alimentos.

El consumo diario de hortalizas en los ECS se encontró mayoritariamente entre 1 ó 2 raciones al día (40,4\% y 42,3\% respectivamente), similar a los EOC, los cuales presentaron su mayor consumo en 2 raciones al día (42,3\%). Sin embargo, se observó una diferencia remarcable en la cantidad de sujetos que no consumían este grupo de alimentos, un 1,9\% de los ECS frente a $9,6 \%$ de los EOC.

Respecto al consumo diario de frutas y zumos naturales, los ECS mostraron su mayor consumo en 3 piezas o más al día (40,4\%), mientras que los EOC en 2 (44,2\%). Un 7,7\% de los ECS no consumían este grupo de alimentos, frente a un $1,9 \%$ de los EOC. Los resultados mostraron, por tanto, un mayor consumo de fruta en los ECS aunque también mayor porcentaje de ECS que no consumían este grupo de alimentos.

Respecto al consumo de carnes rojas, procesadas y embutidos se observó que el $59,6 \%$ de los ECS y el $57,7 \%$ de los EOC consumían una ración de esos alimentos al día. Sin embargo, tan sólo un 19,2\% de los ECS consumían 2 raciones de estos alimentos al día frente al 30,8\% de los EOC.
En referencia al consumo diario de bebidas azucaradas y refrescos, un $78,8 \%$ de los ECS no consumían a diario estos alimentos frente a un $63,3 \%$ de los EOC. Además, Ios consumidores de una unidad al día fueron más en los EOC $(30,8 \%)$, que en los ECS $(19,2 \%)$.

El aceite de oliva fue de consumo diario mayoritariamente alto en los dos grupos de sujetos según lo que evidencia la Tabla 5, siendo de un $82,7 \%$ los ECS que consumen 2 o más raciones al día y de un 76,9\% de los EOC. Sólo un 3,8\% de los ECS y un $5,8 \%$ de los EOC no consumían este alimento diariamente.

Respecto al consumo semanal de pescados y mariscos, el grupo de ECS consumía 1 ración (30,8\%), 2 raciones (32,7\%) o 3 o más raciones (32,7\%). Los EOC presentaron consumos de un $32,7 \%$ para 1 ración, un $34,6 \%$ para 2 raciones y un $23,1 \%$ para 3 o más raciones. Los EOC presentaron por tanto mayores consumos semanales de 1 ó 2 raciones, pero menores consumos en 3 o más raciones, lo cual los alejaba de la buena adherencia a la DM (Tabla 4).

El consumo de legumbres en los ECS estuvo equiparado en 1,2 y 3 raciones o más a la semana con un $30,8 \%, 32,7 \%$ y $32,7 \%$ respectivamente. A diferencia de los EOC que presentaron un consumo diferenciado del $26,9 \%$ para 1 ración, del $48,1 \%$ para 2 raciones y del 19,2\% para 3 o más raciones 
Tabla 4. Alimentación habitual por grupos de alimentos.

\begin{tabular}{|c|c|c|c|c|c|c|c|c|c|c|}
\hline & & & & no suje & (\% suje & & & no sujet & (\% sujet & \\
\hline & & Ración & O R & $1 \mathrm{R}$ & $2 \mathrm{R}$ & $\geq 3 \mathrm{R}$ & OR & $1 \mathrm{R}$ & $2 \mathrm{R}$ & $\geq 3 \mathrm{R}$ \\
\hline & Hortalizas & $100-200 \mathrm{~g}$ & $\begin{array}{c}1 \\
(1,9)\end{array}$ & $\begin{array}{c}21 \\
(40,4)\end{array}$ & $\begin{array}{c}22 \\
(42,3)\end{array}$ & $\begin{array}{c}8 \\
(15,4)\end{array}$ & $\begin{array}{c}5 \\
(9,6)\end{array}$ & $\begin{array}{l}13 \\
(25)\end{array}$ & $\begin{array}{c}22 \\
(42,3)\end{array}$ & $\begin{array}{c}12 \\
(23,1)\end{array}$ \\
\hline 으 & $\begin{array}{l}\text { Frutas y zumos } \\
\text { naturales }\end{array}$ & 1ud. & $\begin{array}{c}4 \\
(7,7)\end{array}$ & $\begin{array}{c}9 \\
(17,3)\end{array}$ & $\begin{array}{c}18 \\
(34,6)\end{array}$ & $\begin{array}{c}21 \\
(40,4)\end{array}$ & $\begin{array}{c}1 \\
(1,9)\end{array}$ & $\begin{array}{c}16 \\
(30,8)\end{array}$ & $\begin{array}{c}23 \\
(44,2)\end{array}$ & $\begin{array}{c}12 \\
(23,1)\end{array}$ \\
\hline 定 & $\begin{array}{l}\text { Carne roja, } \\
\text { procesada y } \\
\text { embutidos }\end{array}$ & $150 \mathrm{~g}$ & $\begin{array}{c}8 \\
(15,4)\end{array}$ & $\begin{array}{c}31 \\
(59,6)\end{array}$ & $\begin{array}{c}10 \\
(19,2)\end{array}$ & $\begin{array}{c}3 \\
(5,8)\end{array}$ & $\begin{array}{c}4 \\
(7,7)\end{array}$ & $\begin{array}{c}30 \\
(57,7)\end{array}$ & $\begin{array}{c}16 \\
(30,8)\end{array}$ & $\begin{array}{c}2 \\
(3,8)\end{array}$ \\
\hline ñ & $\begin{array}{l}\text { Bebidas } \\
\text { carbonatadas/ } \\
\text { azucaradas }\end{array}$ & $200 c c$ & $\begin{array}{c}41 \\
(78,8)\end{array}$ & $\begin{array}{c}10 \\
(19,2)\end{array}$ & $\begin{array}{c}0 \\
(0)\end{array}$ & $\begin{array}{c}1 \\
(1,9)\end{array}$ & $\begin{array}{c}33 \\
(63,5)\end{array}$ & $\begin{array}{c}16 \\
(30,8)\end{array}$ & $\begin{array}{c}2 \\
(3,8)\end{array}$ & $\begin{array}{c}1 \\
(1,9)\end{array}$ \\
\hline & Aceite de oliva & $10-15 g$ & $\begin{array}{c}2 \\
(3,8)\end{array}$ & $\begin{array}{c}7 \\
(13,5)\end{array}$ & $\begin{array}{c}24 \\
(46,2)\end{array}$ & $\begin{array}{c}19 \\
(36,5)\end{array}$ & $\begin{array}{c}3 \\
(5,8)\end{array}$ & $\begin{array}{c}9 \\
(17,3)\end{array}$ & $\begin{array}{l}26 \\
(50)\end{array}$ & $\begin{array}{c}14 \\
(26,9)\end{array}$ \\
\hline 光 & $\begin{array}{l}\text { Pescados y } \\
\text { mariscos }\end{array}$ & $150-200 \mathrm{~g}$ & $\begin{array}{c}2 \\
(3,8)\end{array}$ & $\begin{array}{c}16 \\
(30,8)\end{array}$ & $\begin{array}{c}17 \\
(32,7)\end{array}$ & $\begin{array}{c}17 \\
(32,7)\end{array}$ & $\begin{array}{c}5 \\
(9,6)\end{array}$ & $\begin{array}{c}17 \\
(32,7)\end{array}$ & $\begin{array}{c}18 \\
(34,6)\end{array}$ & $\begin{array}{c}12 \\
(23,1)\end{array}$ \\
\hline$\sum_{u}^{z}$ & Legumbres & $150 \mathrm{~g}$ & $\begin{array}{c}2 \\
(3,8)\end{array}$ & $\begin{array}{c}16 \\
(30,8)\end{array}$ & $\begin{array}{c}17 \\
(32,7)\end{array}$ & $\begin{array}{c}17 \\
(32,7)\end{array}$ & $\begin{array}{c}3 \\
(5,8)\end{array}$ & $\begin{array}{c}14 \\
(26,9)\end{array}$ & $\begin{array}{c}25 \\
(48,1)\end{array}$ & $\begin{array}{c}10 \\
(19,2)\end{array}$ \\
\hline 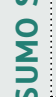 & Frutos Secos & $30 \mathrm{~g}$ & $\begin{array}{c}10 \\
(19,2)\end{array}$ & $\begin{array}{c}11 \\
(21,2)\end{array}$ & $\begin{array}{c}6 \\
(11,5)\end{array}$ & $\begin{array}{c}25 \\
(48,1)\end{array}$ & $\begin{array}{c}14 \\
(26,9)\end{array}$ & $\begin{array}{c}15 \\
(28,8)\end{array}$ & $\begin{array}{c}12 \\
(23,1)\end{array}$ & $\begin{array}{c}11 \\
(21,2)\end{array}$ \\
\hline 어 & $\begin{array}{l}\text { Bollería y } \\
\text { repostería } \\
\text { industrial }\end{array}$ & 1ud. & $\begin{array}{c}13 \\
(25)\end{array}$ & $\begin{array}{c}16 \\
(30,8)\end{array}$ & $\begin{array}{c}6 \\
(11,5)\end{array}$ & $\begin{array}{c}17 \\
(32,7)\end{array}$ & $\begin{array}{c}9 \\
(17,3)\end{array}$ & $\begin{array}{c}10 \\
(19,2)\end{array}$ & $\begin{array}{c}15 \\
(28,8)\end{array}$ & $\begin{array}{c}18 \\
(34,6)\end{array}$ \\
\hline
\end{tabular}

ECS: Estudiantes de Ciencias de la Salud; EOC: Estudiantes de otras ciencias; R: raciones.

semanales. Por lo que se concluye que los EOC presentaron consumos bajos de este grupo de alimentos (Tabla 4).

En los frutos secos, la Tabla 4 indica que un $48,1 \%$ de los ECS consumían 3 o más raciones semanales, mientras que sólo un $21,2 \%$ de los EOC presentaron ese consumo. Los resultados en los EOC muestran que la mayoría de sujetos no consumen nada $(26,9 \%)$ o sólo 1 ración semanal $(28,8 \%)$, por lo que el consumo de frutos secos sería insuficiente en este grupo.

Finalmente, respecto al consumo de bollería y repostería industrial presentado en la Tabla 4, el 32,7\% de los ECS tuvieron un consumo de 3 o más raciones semanales, siguiéndole un $30,8 \%$ en el consumo de 1 unidad semanal. En los EOC, el consumo mayoritario $(34,6 \%)$ también se encontró en 3 o más unidades por semana, seguido en este caso por el consumo de 2 unidades (28,8\%). Los mayores porcentajes de consumo en este grupo de alimentos están en 3 o más porciones semanales tanto en ECS como en EOC, datos muy desfavorables para la adherencia a la DM.

\section{DISCUSIóN}

La literatura que encontramos sobre los hábitos de vida de universitarios suele poner de manifiesto que la mayoría de universitarios/as no practican hábitos saludables durante los años que duran los estudios ${ }^{22}$. Sin embargo, la carencia de investigaciones que comparen grados universitarios que tengan que ver con la salud y otros que no tengan nada que ver, justifica el presente estudio.

En nuestro estudio se puede observar cómo la mayor parte de la población universitaria participante no mantiene, en general, buenos hábitos saludables. Estos resultados 
concuerdan con los de una revisión de vida saludable en universitarios que reportó que los jóvenes consumen dietas desequilibradas con alto contenido calórico y donde la práctica de AF es insuficiente o nula ${ }^{22}$. Sí es cierto que respecto a la $A F$, los ECS practican mayor cantidad de AF moderada e intensa, la cual conduce al organismo hacia una amplia diversidad de mejoras para la salud ${ }^{23}$. Según la literatura24,25, el entrenamiento de alta intensidad produce más mejoras a nivel muscular y cardiorrespiratorio -previniendo enfermedades cardiovasculares- que el de menor intensidad. También se asocia a un mejor control de la glucosa en sangre, previniendo el riesgo de diabetes tipo II. Hay que tener en cuenta, sin embargo, que los resultados podrían tener un componente subjetivo alto, ya que la población tiende a sobrestimar la intensidad de las actividades físicas leves o moderadas ${ }^{26}$. Respecto a los EOC, la práctica mayoritaria de AF es leve. Esta situación se produce sobre todo en época de exámenes, ya que pasan inactivos las horas de estudio debido a toda la carga y el estrés que soportan durante esos periodos. Flores et al. ${ }^{27}$ reportaron que la mayor barrera que los estudiantes encontraban para la práctica de AF era la falta de tiempo. Esta situación se ha dado en el presente estudio, ya que los datos fueron recogidos durante mayo y junio, meses en los que los universitarios dedican mucho tiempo a estudiar para sus evaluaciones finales. Por otro lado, otros autores afirman que el practicar AF en periodos de mucho estrés, como los de evaluación, permite al estudiante rendir más y mejor e incluso obtener mejores notas $^{28,29}$. Así, la regularidad en la AF que mantiene el grupo de ECS favorecería no sólo su salud, sino también sus resultados académicos.

Cabe que destacar que en el presente estudio, los datos sobre el total de sujetos de cada grupo que practicaban más AF de intensidad leve, moderada o vigorosa, se obtuvieron meticulosamente y de acuerdo con a la metodología del cuestionario IPAQ. Según Pérez et $a l^{30}$, este cuestionario se debería usar con fines descriptivos y definidores del comportamiento global de una población, pero no a la hora de definir la práctica de la AF con una mayor precisión.

Respecto a los hábitos alimentarios, los resultados indican que no son muy acordes a una rutina alimentaria saludable, ya que de entre el total de la población del estudio, sólo un 40,4\% presentó adherencia a la DM. Otras investigaciones han reportado adherencias de sólo el 33\% de los participantes ${ }^{31}$ o incluso de tan sólo el $26 \%{ }^{32}$, situación alarmante cuando la cantidad de sujetos que participaron en estos estudios fueron de 266 y 490, respectivamente. Existen estudios con 614 estudiantes que reportan incluso adherencias tan bajas como del $12 \%$ en mujeres y del $9 \%$ de hombres ${ }^{33}$. Si estos datos los comparamos con la presente investigación, vemos que la adherencia resultante no es tan baja (en los ECS es del $54 \%$ y en los EOC del $27 \%$ ). Vega et al..$^{34}$ compararon estudiantes de enfermería con otros de educación, dándoles una formación específica de nutrición durante un período de tiempo de un año. Pese a eso, de los 399 sujetos partícipes del estudio, un $57 \%$ seguían sin presentar adherencia a la DM, siendo además los enfermeros/as los que mantuvieron peores hábitos nutricionales. En nuestro estudio, los ECS sí reportan mejores hábitos saludables que los EOC.

Respecto al consumo de los grupos de alimentos destaca que el consumo de hortalizas está mayoritariamente situado entre 1 y 2 raciones al día en los dos grupos de estudiantes (ECS y EOC), por lo que es insuficiente según las directrices de una dieta saludable ${ }^{21}$. Las hortalizas son una fuente principal de vitaminas hidrosolubles, antioxidantes y fibra, cuyo consumo está científicamente comprobado que previene la aparición de enfermedades ${ }^{35}$. Varias investigaciones clínicas han concluido que el consumo de estos alimentos contribuye a la mejora de la función cerebral, ayudan a disfrutar de un envejecimiento saludable y frenan el desarrollo de enfermedades neurodegenerativas. Además, numerosos estudios epidemiológicos han reportado una relación inversa entre el consumo de verduras y los problemas de salud crónicos como el cáncer y las enfermedades cardiovasculares y neurodegenerativas ${ }^{36}$.

En lo relativo al consumo de frutas, debería ser diario y de 2 o más piezas al día. Los resultados de la investigación muestran que un 40,4\% de los ECS consumen 3 o más unidades al día, mientras que los EOC tienen su mayor consumo en 1-2 piezas; por lo que los ECS sí cumplen con las recomendaciones de la DM. Las frutas son una fuente importante de antioxidantes y fibra. Su consumo también beneficia a las funciones cerebrales y cardiovasculares, además de contribuir a la prevención del cáncer ${ }^{36}$. En un estudio realizado en Zamora se obtuvieron resultados de patrones de consumo en los jóvenes estudiantes muy por debajo de las recomendaciones respecto al consumo de frutas ${ }^{37}$. Esta situación no favorece en absoluto el mantenimiento de la salud a largo plazo, ni la conservación de hábitos saludables en la edad adulta.

Respecto al consumo de aceite de oliva, sí representa la principal fuente de grasa para la mayoría de los estudiantes partícipes del estudio. Un 92,3\% de los sujetos totales lo tiene como preferencia a la hora de cocinar o como aliño principal. Pascual et al. ${ }^{38}$ realizaron un estudio a universitarios de ciencias de la salud y concluyeron que el consumo de aceite de oliva es preferente además de ser consumido 2 o más veces al día. En el presente estudio, alrededor del $86 \%$ de los ECS lo consumen en 2 ó 3 raciones diarias. Los EOC tienen un consumo ligeramente más bajo, aproxima- 
damente un $77 \%$. Sin embargo, los dos grupos lo consumen de forma habitual y suficiente, por lo que los resultados son favorables al respecto.

Por el contrario, el consumo de carne roja, carne procesada y embutidos dista mucho de ser el óptimo. Las recomendaciones afirman que 1 ración o menos a la semana sería lo recomendado ${ }^{21}$. Los resultados muestran que un $78,8 \%$ de los ECS y un $88,5 \%$ de los EOC consumen de 1 a 2 raciones al día. A estos datos se suma que un $5,8 \%$ de los ECS y un $3,8 \%$ de los EOC consumen 3 o más raciones de estos alimentos al día. Según diversas fuentes, el consumo excesivo de proteína animal procedente de carnes rojas o procesadas aumenta el riesgo de padecer cáncer, diabetes mellitus tipo II e hipercolesterolemia ${ }^{39-40}$. Sin embargo, cabe destacar que, si la carne roja no es procesada y es magra, la cual es baja en grasas saturadas, en un consumo equilibrado y combinada con productos saludables como las verduras y las frutas, su consumo está asociado a reducciones en el colesterol LDL en adultos sanos y en adultos con hipercolesterolemia. Además, es una fuente de proteína de alto valor biológico, vitamina B12, zinc y hierro ${ }^{41}$.

El consumo de bollería y repostería industrial representa un aporte significativo de carbohidratos refinados y azúcares simples a la dieta ${ }^{35}$. Según Valle et al. ${ }^{42}$, el consumo de este tipo de alimentos representa un incremento significativo en el desarrollo de enfermedades como la diabetes tipo II, la obesidad y problemas cardiovasculares. El aumento de los niveles de triglicéridos y glucosa en sangre debido a estos alimentos conduce a cambios metabólicos y hormonales que estimulan la sensación de hambre y promueven la acumulación de grasa, favoreciendo así enfermedades como la obesidad. Los resultados de la presente investigación resultan en altos consumos de bollería y repostería industrial. Un $32,7 \%$ de los ECS consumen 3 o más unidades de estos productos a la semana y un $42,3 \%$ consumen entre 1 y 2 unidades. Con respecto a los EOC, son un $34,6 \%$ los que consumen 3 o más porciones y un $48 \%$ entre 1 y 2 . Por ello, un $53,8 \%$ de la totalidad de estudiantes encuestados rebasan las recomendaciones de la OMS a este respecto ${ }^{43}$. Latasa et al. ${ }^{44}$ demostraron que el consumo de estos alimentos y el aumento de los azúcares refinados que contienen se han triplicado entre 1990 y 2010 en la población española, por lo que la población consume tres veces más de estos productos con el triple de azúcares que en años previos.

Respecto al consumo de pescado y marisco, los ECS coinciden en un $32,7 \%$ de sujetos que comen 2 raciones o 3 o más a la semana. Los EOC, en cambio, reportan un $34,6 \%$ en el consumo de 2 raciones y un 23,1\% en 3 raciones o más. La recomendación está entre 2 y 4 veces por semana ${ }^{21}$, por lo que más de la mitad, tanto de los EOC como de los ECS, pre- sentan buenos hábitos en cuanto al consumo de pescado. El pescado y el marisco son una fuente de proteínas, sales minerales, vitaminas del grupo $B$, omega- 3 y gran cantidad de agua ${ }^{35}$, por lo que su consumo habitual representa un aporte nutricional de calidad. Pese a ello, Alegria et al. ${ }^{45} \mathrm{com}$ pararon el consumo de carne y pescado, y concluyeron que un $91,5 \%$ de los universitarios españoles consumían más carne que pescado habitualmente. Asimismo, De Piero et al. ${ }^{46}$ concluyeron que el consumo medio total de 329 estudiantes universitarios era de 0,3 piezas de pescado/marisco por semana. Y finalmente, Redondo et al. ${ }^{47}$ realizó un estudio con universitarios deportistas y no deportistas que resultó en consumos de pescados y mariscos de entre 1,5 y 2 raciones por semana. Si comparamos los resultados de las investigaciones con el presente estudio, se puede observar que el consumo de este grupo de alimentos en nuestro estudio es mayor en los dos grupos de sujetos, resultando en un consumo ligeramente mayor en el grupo de los ECS.

En lo que respecta al consumo de legumbres, los resultados también se encuentran por debajo de las recomendaciones de 3 raciones por semana ${ }^{21}$. De los ECS un 63,5\% sólo come legumbres 1 ó 2 veces por semana y de los EOC un 75\%. Y sólo encontramos un $32,7 \%$ de ECS y un $19,2 \%$ de EOC que cumplan con las recomendaciones de 3 o más veces por semana. Las legumbres son una fuente de proteínas e hidratos de carbono complejos. A parte, contienen vitaminas del grupo $B$ mayoritariamente, minerales como el calcio y el hierro y un contenido considerable de fibra ${ }^{35}$. Según De Piero et al. ${ }^{46}$, los cuales recogieron el consumo habitual de los grupos de alimentos en 329 estudiantes universitarios, el consumo de legumbres no sobrepasaba las 0,21 raciones semanales. Martínez et al..$^{48}$ estudiaron las preferencias de consumo de alimentos en el comedor de una universidad española y uno de los platos menos elegidos fueron las legumbres con un consumo del 9,8\%. Amo et al. ${ }^{49}$ estudiaron los hábitos nutricionales de la población universitaria en base a la compra de alimentos en el supermercado de la universidad. Los resultados dejaron ver que las legumbres, a pesar de ser más baratas, son de las menos elegidas en la cesta de la compra de los estudiantes y por lo tanto, las menos consumidas.

Los frutos secos son ricos en vitaminas, fibra, minerales y poseen un alto contenido de ácidos grasos insaturados. Los principales beneficios de la ingesta de éstos son los efectos que tienen a la hora de prevenir el cáncer o las enfermedades cardiovasculares ${ }^{50}$. Según Urquiaga et al. ${ }^{21}$, una ingesta adecuada debería sobrepasar las 3 veces por semana. Los resultados en nuestro estudio muestran que un 48,1\% de Ios ECS sí cumplen con las recomendaciones al contrario que los EOC, la mayoría de los cuales $(55,7 \%)$ los consumen 
entre 0 y 1 vez por semana. Pinto de Souza et al. ${ }^{51}$ trabajaron con estudiantes de enfermería, nutrición y medicina, y concluyeron que los enfermeros/as sí cumplían con las recomendaciones de consumo en este grupo de alimentos, coincidiendo con el presente estudio. Asimismo, Sánchez et al. ${ }^{52}$ realizaron un estudio a ECS y reportaron un aumento en el consumo de frutos secos del primer curso al segundo curso del grado, debido a los conocimientos que adquirieron los estudiantes con respecto a buenos hábitos alimentarios.

Tanto los ECS como los EOC reflejan un consumo bajo de verduras, hortalizas y legumbres, que podría conducir a carencias de micronutrientes. El consumo de fruta y frutos secos está por debajo del recomendado, pero tiene mejores resultados. Con respecto al consumo de pescados y mariscos se obtienen resultados bajos frente al consumo excesivo de carnes, cosa que les aportaría más ácidos grasos saturados que insaturados. Los carbohidratos simples y azúcares presentan un porcentaje de consumo elevado, por lo que puede haber un riesgo futuro en el desarrollo de enfermedades relacionadas con el metabolismo de la glucosa.

Por grupos de estudio, los ECS reportan una mayor adherencia a la DM, con consumos adecuados respecto a frutas, bebidas azucaradas, aceite de oliva y frutos secos. Los consumos aceptables los encontramos en verduras, hortalizas y legumbres, e inadecuados en carnes procesadas, embutidos y bollería industrial. En referencia a la práctica de la AF moderada e intensa es un hábito presente en casi todos los sujetos del estudio.

El grupo de EOC reporta baja adherencia a la DM, siendo un factor negativo para la misma según muestra el análisis de regresión, y con resultados bajos en el consumo de frutas, legumbres y frutos secos; consumos aceptables en verduras y hortalizas, pescados y mariscos y bebidas azucaradas; altos en carnes procesadas y embutidos y bollería industrial. Y finalmente, un consumo elevado de aceite de oliva. Respecto a la práctica de $A F$, se reporta menos práctica habitual y de menor intensidad que en el grupo de ECS.

Se concluye, por tanto, que un poco más de la mitad de los ECS reportan hábitos nutricionales correctos en concordancia con su adherencia a la DM. Por otro lado, sí que presentan buenos y amplios hábitos de práctica de AF.

En referencia a los EOC, menos del 30\% reporta hábitos nutricionales adecuados que respondan a una buena adherencia a la DM. Y respecto a la AF, la práctica es baja y de intensidad leve asociada principalmente a los desplazamientos de la vida diaria y no a la práctica de ejercicio o deporte voluntario.
En futuras investigaciones, se podría hacer un seguimiento de los hábitos nutricionales de los universitarios estudiados durante todos los años del grado, y ver si realmente los conocimientos que reciben les llevan a prácticas de vida más saludables. Asimismo, podrían extraerse líneas de investigación futuras para ver cuáles son las ramas de conocimiento donde se tienden a practicar peores hábitos saludables. $Y$ finalmente, se recomienda intentar utilizar otro cuestionario validado u otros aparatos como el acelerómetro para ser más precisos en la medición de la AF, ya que el protocolo de obtención de resultados no es el adecuado para clasificar intensidades. La AF debería diferenciarse por intensidades para poder evaluar sus beneficios, ya que según la literatura los cambios a nivel del metabolismo o del sistema muscular, por ejemplo, son diferentes. También se sugiere un instrumento de medición objetivo para que la población valore las intensidades a las que practica AF, ya que se sabe que son subjetivas y tienden a sobrestimarlas.

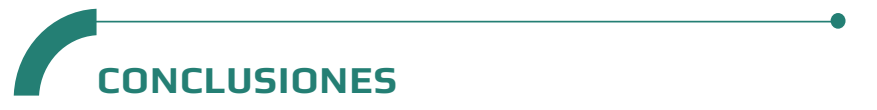

Los ECS sí reportan más adherencia a la DM y mejores hábitos nutricionales que los EOC, así como una mayor práctica de AF y de mejor calidad para la mejora de parámetros relacionados con la salud y calidad de vida. Se demuestra así una relación directa entre los conocimientos en salud y unos mejores hábitos saludables, una mayor práctica regular de $\mathrm{AF}$ vigorosa y una mayor adherencia a la DM.

\section{CONTRIBUCIÓN DE AUTORÍA}

AS fue la responsable de la recogida de datos, análisis y redacción del manuscrito.

AG participó en la supervisión y dirección del estudio, así como en el análisis estadístico y la redacción del manuscrito. Todos los autores han revisado y aceptado la versión final del mismo.

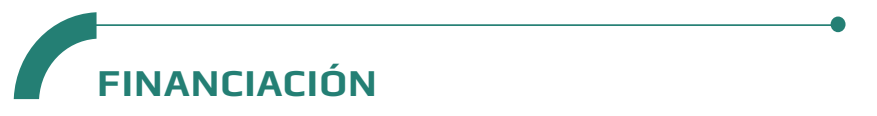

Ninguna entidad financiadora ha participado en la ejecución de la investigación. 


\section{CONFLICTO DE INTERESES}

Las autoras expresan que no existen conflictos de interés al redactar el manuscrito.

\section{REFERENCIAS}

(1) Pérez IJ, Rivera E, Delgado M. Mejora de hábitos de vida saludables en alumnos universitarios mediante una propuesta de gamificación. Nutr Hosp. 2017; 34(4): 942-51.

(2) González G, Padial R, Espejo T, Chacón R, Puertas P, Pérez AJ. Relación entre clima motivacional hacia el deporte y adherencia a la dieta mediterránea en estudiantes universitarios de educación física. Int ] Develop \& Educ Psychol INFAD. 2017; 4(1): 285-95.

(3) Escámez JC, Gálvez A, Gómez L, Escribá AR, Tárraga P, Tárraga L. Influencia de la actividad física y la capacidad aeróbica sobre el rendimiento académico en la adolescencia: una revisión bibliográfica. JONNPR. 2018; 3(1): 49-64.

(4) Cardarso A, Dopico X, Iglesias E, Cadarso CM, Gude F. Calidad de vida relacionada con la salud y su relación con la adherencia a la dieta mediterránea y la actividad física en universitarios de Galicia. Nutr Clin Diet Hosp. 2017; 37(2): 42-9.

(5) Serra L, Aranceta J. Dieta Mediterránea: una visión global. La nutrición comunitaria en el siglo XXI. 1a ed. Barcelona: Real Academia Europea de Doctores; 2017.

(6) Márquez F, Bulló B, Vizmanos B, Casas P, Salas J. Un patrón de alimentación saludable: la dieta mediterránea tradicional. Antropo. 2008; 16: 11-22.

(7) Cuervo C, Cachón J, Zagalaz ML. Hábitos alimentarios y práctica de actividad física en adolescentes. J Sport Health Res. 2017; 9(1): 75-84.

(8) Jiménez MC, Sanabria MC, Mendoza L, González R. Factores de riesgo cardiovascular en escolares y adolescentes de una comunidad rural de Amambay. Rev Pediatr (Asunción). 2011; 38(3): 205-12.

(9) Kovesdy CP, Furth S, Zoccali C. Obesidad y enfermedad renal: consecuencias ocultas de la epidemia. Nephrol. 2017; 37(4): 360-9.

(10) Ping Y, Wienert J, Hu C, Yan G, Lippke S. Web-Based Intervention for Physical Activity and Fruit and Vegetable Intake Among Chinese University Students: A Randomized Controlled Trial. J Med Internet Res. 2017; 19(4): e106.

(11) González L, Carreño C, Estrada A, Monsalve J, Stella L. Exceso de peso corporal en estudiantes universitarios según variables sociodemográficas y estilos de vida. Rev Chil Nutr. 2017; 44(3): 251-61.

(12) Joyce J, Gitomer D, Laconangelo C. Classrom assignements as measures of teaching quality. Learn \& Instruc. 2018; 54: 48-61.

(13) Ríos MR, Rodríguez N. Estilo de vida, autoconcepto y trastornos emocionales en estudiantes universitarios obesos. Rev Elect Psicol Iztacala. 2017; 20(3): 1009-25.

(14) García-Laguna DG, García-Salamanca GP, Tapiero YT, Ramos
DM. Determinantes de los estilos de vida y su implicación en la salud de jóvenes universitarios. Hacia Prom Salud. 2012; 17(2): 169-85.

(15) Muñoz G, Lozano MC, Romero CS, Pérez J, Veiga P. Evaluación del consumo de alimentos de una población de estudiantes universitarios y su relación con el perfil académico. Nutr Hosp. 2017; 34(1): 134-43.

(16) Cancela JM, Ayán C. Prevalencia y relación entre el nivel de actividad física y las actitudes alimenticias anómalas en estudiantes universitarias españolas de ciencias de la salud y la educación. Rev Esp Salud Pública. 2011; 85(5): 499-505.

(17) Gresse A, Steenkamp L, Pietersen J. Eating, drinking and physical activity in Faculty of Health Science students compared to other students at a South African university. South Afr ] Clin Nutr. 2015; 28(4): 154-9.

(18) Manchola J, Bagur C, Girabent M. Fiabilidad de la versión española del cuestionario de actividad física PAQ-C. Rev Int Med Cienc Act Fis Dep. 2017; 17(65): 139-52.

(19) Papadaki A, Johnson L, Toumpakari Z, England C, Toms S, Penfold $C$, et al. Validation of the English Version of the 14item Mediterranian Diet Adherence Screener of the PREDIMED Study, in People al High Cardiovascular Risk in the UK. Nutrients. 2018; 10(2): 138.

(20) Rodríguez T, Fernández J, Cucó G, Biarnés E, Arija V. Validación de un cuestionario de frecuencia de consumo alimentario corto: reproducibilidad y validez. Nutr Hosp. 2008; 23(3): 242-52.

(21) Urquiaga I, Echevarría G, Dussaillant C, Rigotti A. Origen, componentes y posibles mecanismos de acción de la dieta mediterránea. Rev Med Chil. 2017; 145(1): 85-95.

(22) Sánchez MA, De Luna E. Hábitos de vida saludable en la población universitaria. Nutr Hosp. 2015; 31(5): 1910-9.

(23) Práxedes A, Sevil J, Moreno A, Del Villar F, García, L. Niveles de actividad física en estudiantes universitarios: diferencias en función del género, la edad y los estados de cambio. Rev Iber Psicologia Ejer Depor. 2016; 11(1): 123-32.

(24) Mandrup CM, Egelund J, Nyberg MP, Slingsby MH, Andersen $C B$, Logstrup $S$, et al. Effects of high-intensity training on cardiovascular risk factors in pre-and postmenopausal women. JOGN Nurs. 2017; 216(4).

(25) Cassidy S, Thoma C, Houghton D, Trenell M. High-intensity interval training: a review of its impact on glucose control and cardio metabolic health. Diabetologia. 2017; 60(7): 7-23.

(26) Renato F. Frecuencia de práctica y percepción de la intensidad de las actividades físicas más frecuentes en adultos. Rev PanAmaz Saude. 2010; 1(3): 61-7.

(27) Flores G, Ruiz F. Motivaciones de los estudiantes universitarios para nunca realizar actividades físico-deportivas de tiempo libre. El caso de la Universidad de Guadalajara. Nuev Tend Ed Fís Dep y Recreac. 2010; 17: 34-7.

(28) Wunsch K, Kasten N, Fuchs R. The effect of physical activity on sleep quality, well-being and affect in academic stress periods. Nat Sci Sleep. 2017; 9: 117-26.

(29) Crozier AJ, Spink K. Effect of Manipulating Descriptive Norms and Positive Outcome Expectations on Physical Activity of University Students during Exams. ] Health Commun. 2017; 32(6): 784-90.

(30) Pérez J], García E. Medición de la Actividad Física Mediante el International Physical Activity Questionaire (IPAQ) en Estudios 
Españoles e Internacionales. Rev Elect Act Fis Cien. 2012; 4(2): 1-21.

(31) De la Montaña J, Cobas N, Rodríguez M, Míguez M. Adherencia a la dieta mediterránea y su relación con el índice de masa corporal en universitarios de Galicia. Nutr Clin Diet Hosp. 2012; 32(3): 72-80.

(32) Chacón R, Castro M, Muros J], Espejo T, Zurita F, Linares M. Adhesión a la dieta mediterránea en estudiantes universitarios y su relación con los hábitos de ocio digital. Nutr Hosp. 2016; 33(2): 405-10.

(33) Congreso Iberoamericano de Universidades Promotoras de la Salud (CIUPS 2017): Promoción de la Salud y Universidad. Construyendo Entornos Sociales y Educativos Saludables. Univ. Alicante (España), 27-29 junio 2017.

(34) Vega MR, Ejeda JM, González MP, Mijancos MT. Cambios en la adherencia a la dieta mediterránea en estudiantes de los Grados de Enfermería y de Magisterio tras cursar una asignatura de nutrición. Nutr Hosp. 2014; 30(5): 1173-80.

(35) Condeminas C, Díaz-Guerra A. Bioquímica i fisiología de la nutrició. Apuntes académicos UOC. 2014.

(36) Yahia EM. Fruit and Vegetable Phytochemicals: Chemistry and Human Health. 2a ed. USA: John Wiley \& Sons Ltd; 2018.

(37) Pastor R, Bibiloni MM, Tur Mari JA. Patrones de consumo de alimentos en estudiantes universitarios de Zamora. Nutr Hosp. 2017; 34(6): 1424-31.

(38) Pascual SA. Frecuencia de consumo de alimentos en estudiantes universitarios. Trabajo de Final de Grado. León: Universidad de León; 2017.

(39) Rodríguez J. Nutrición y Cáncer. Trabajo de Final de Grado. Facultad de farmacia; 2017.

(40) Elmadfa I, Meyer A. Animal Proteins as Important Contributors to a Healthy Human Diet. Annu Review Anim Biosci. 2017; 5: 111-31.

(41) Li D, Sirithon S, Wahlqvist M, Mann N, Sinclair A. Lean meat and heart health. Asia Pac J Clin Nutr. 2005; 14(2): 113-9.

(42) Valle AG. Creación de una línea de productos de bollería para diabéticos del centro de salud no 2 ubicado en Cantón Ambato.
Trabajo de Final de Grado. Univ. Regional Andes, Argentina; 2017.

(43) Ruiz E, Varela G. Adecuación de la ingesta de azúcares totales y añadidos en la dieta española a las recomendaciones: estudio ANIBES. Nutr Hosp. 2017; 34(4): 45-52.

(44) Latasa P, Louzada ML, Martinez E, Monteiro CA. Added sugars and ultra-processed foods un Spanish households (19902010). Eur J Clin Nutr. 2017; 72(6).

(45) Alegria I, Rocandio AM, Telletxea S, Rincón E, Arroyo M. Relación entre el índice de consumo de pescado y carne y la adecuación y calidad de la dieta en mujeres jóvenes universitarias. Nutr Hosp. 2014; 30(5): 1135-43.

(46) De Piero A, Bassett N, Rossi A, Sammán N. Tendencia en el consumo de estudiantes universitarios. Nutr Hosp. 2015; 31(4): 1824-31.

(47) Redondo MP, De Mateo B, Carreño L, Marugán JM, Fernández M, Camina MA. Ingesta dietética y adherencia a la dieta mediterránea en un grupo de estudiantes universitarios en función de la práctica deportiva. Nutr Hosp. 2016; 33(5): 1172-8.

(48) Martínez JR, García R, Villarino A, Marrodán MD, Serrano L. Eating Habits and preferences among the student population of the Complutense University of Madrid. Public Health Nutr. 2015; 18(14): 2654-9.

(49) Amo E, Escribano F, Gracia MJ, Pardo I. Are eating habits of university students different to the rest of the Spanich population? Food availability, consumption and cost. Span J Agric Res. 2016; 14(2): e0103.

(50) Grosso G, Yang J, Marventano S, Micek A, Galvano F, Kales S. Nut consumption on all-cause, cardiovascular, and cáncer mortality risk: a systematic review and meta-analysis of epidemiologic studies. Am J Clin Nutr. 2015; 101(4): 783-93.

(51) Pinto de Souza D, Lopes L, Gomes EC, Queiroz A, Lopes MS. Food consumption in Students of the Health Area of Brazilian Public University. Food Public Health. 2016; 6(2): 38-43.

(52) Sánchez MA, Gutiérrez A, Rizo M, Cortés E. Estudio longitudinal sobre la adherencia al patrón de Dieta Mediterránea en estudiantes de Ciencias de la Salud. Rev Esp Nutr Hum Diet. 2018; 22(1): 4-13. 